\title{
Sacral neuromodulation: emerging technology with expanding indications
}

\author{
M. M. Karram
}

Published online: 23 October 2010

(C) The International Urogynecological Association 2010

Sacral neuromodulation was first described in 1981 by Tanagho and Schmidt as a potential bladder pacemaker in which pulsatile electrical stimuli are delivered to the S3 sacral nerve roots, thereby regulating many of the functions of the pelvic floor neural network. Since its approval as a medical device in 1994 in Europe and in 1997 in the USA, sacral neuromodulation has been used for a variety of urinary tract disorders, chronic pain syndromes, and more recently defecatory dysfunction. While the mechanism of action of the device continues to be poorly understood, there is no question that this has been a welcome intervention to the armamentarium of the pelvic floor specialist, as it addresses many areas of function that have historically been very poorly managed.

This issue of our journal is accompanied by a supplement dedicated to sacral neuromodulation. Under the direction of guest editor Dr. Charles Butrick, thought leaders with extensive experience with this device have been invited to write review articles on numerous aspects of neuromodulation. Topics discussed include the history of the device, the proposed mechanism of action, appropriate patient selection, procedural techniques, neurodiagnostics, clinical outcomes for urge incontinence, and retention, as well as how best to troubleshoot complications of the device. Furthermore, three accompanying review articles are presented in this issue of the journal that address current off-label indications in the USA. These include utilization of the device for fecal incontinence, interstitial cystitis, chronic pelvic pain, and sexual dysfunction, as well as alternative approaches to sacral neuromodulation. I want to thank Dr. Butrick and all the invited contributors as I am sure this up-to-date review will be very well received by our readership and by our subspecialty as a whole.

M. M. Karram $(\bowtie)$

Urogynecology, The Christ Hospital,

2123 Auburn Ave. Suite 307,

Cincinnati, OH 45219, USA

e-mail: Mickey.Karram@thechristhospital.com 